\title{
ENDOSCOPIC VEIN HARVEST FOR CORONARY ARTERY BYPASS GRAFTING: TECHNIQUE AND OUTCOMES
}

Z. Davis, MD ${ }^{\mathrm{a}, \mathrm{b}}$

H. Kurt Jacobs, $\mathrm{PhD}^{\mathrm{b}, \mathrm{c}}$

Mark Zhang, $\mathrm{PA}^{\mathrm{a}}$

Celia Thomas, RN, $\mathrm{MS}^{\mathrm{a}}$

Yolanda Castellanos, $\mathrm{BS}^{\mathrm{b}}$
Background: The greater saphenous vein is a common conduit for coronary revascularizations. Traditional vein harvesting uses long incision(s) that can lead to significant morbidities. A minimally invasive technique has been developed that allows the harvest of much of the saphenous vein with one incision and fewer morbidities. Methods: Our technique and outcomes on 110 patients with minimally invasive harvest (endoscopic vein harvesting) is presented. Comparisons are made with an equivalent retrospective group within the same hospital and to a smaller $(n=28)$ prospective group at other hospitals. Results: Endoscopic vein harvesting has evolved to one above-knee incision of $3 \mathrm{~cm}$ length that allows for the harvest of $35 \mathrm{~cm}$ of vein. Harvest times were longer for endoscopic vein harvesting, showed a learning curve, and appeared to reach a baseline of 35 minutes. Incision closure times were less for the endoscopic vein harvesting group. Total skin to skin operating times for the entire cardiovascular procedure did not differ between the groups. In relatively homogeneous populations, leg infection rates did not differ, but other leg morbidities were less for the patients who underwent endoscopic vein harvesting. Hospital readmissions for leg wound care were low in both groups although the number of office visits required for leg care was higher for patients undergoing traditional vein harvesting. Pain perception by the patients was much less for the endoscopic vein harvesting and remained lower for up to 4 weeks. Conclusions: Although endoscopic vein harvesting is a relatively new procedure, it is safe, effective, and less painful for the patient and carries fewer morbidities. (J Thorac Cardiovasc Surg 1998;116:228-35)
$T^{\mathrm{r}}$ he current trend toward less invasive cardiothoracic surgery is logically extended to minimally invasive vein harvesting because most coronary ar-

From The Edward Cardiovascular Institute, Edward Hospital, and the Cardiac Surgery Associates, ${ }^{\text {a }}$ Naperville, Ill., The Institute for Minimally Invasive Surgery, ${ }^{\mathrm{b}}$ West Chicago, Ill., and Research Service, Hines Veterans Administration Hospital, ${ }^{\mathrm{c}}$ Hines, Ill.

This work was supported in part by funding to The Institute for Minimally Invasive Surgery from Central DuPage Hospital, Winfield, Ill., and from Ethicon Endo-Surgery, Cincinnati, Ohio.

This work was performed at Edward Cardiovascular Institute, Naperville, Ill.

Received for publication August 25, 1997; revisions requested Nov. 12, 1997; revisions received April 2, 1998; accepted for publication April 3, 1998.

Address for reprints: H. K. Jacobs, PhD, 550 E. Washington, West Chicago, IL 60185.

12/1/90840 tery bypass grafting (CABG) uses a segment of saphenous vein. ${ }^{1}$ Although infrequently reported, ${ }^{2}$ patient complications and/or delayed healing of the harvest site often dominate the recuperative phase of CABG. ${ }^{3}$ Indeed, with a broad definition for leg complications, the complication rate has been reported from $24.3 \%{ }^{4}$ to $43.8 \% .^{5}$ Endoscopic vein harvesting $(\mathrm{EVH})$, although still an evolving technique, ${ }^{6-9}$ may significantly alter this rate and provide greater patient acceptance.

\section{Methods}

Prospective data were taken during our first 8-month experience with EVH for CABG. There were 121 consecutive patients in this series. Any patient undergoing another concurrent cardiac or vascular procedure was excluded from study. Three additional patients with a mixed harvest (EVH for thigh, traditional harvesting for calf) were also excluded. Patients with cardiogenic shock $(n=1)$, used as a strong contraindication for EVH, were also excluded. This resulted in $110 \mathrm{EVH}$ cases. These data 
were compared with a retrospective group of the immediately preceding 121 consecutive traditional harvest cases (traditional) at the same hospital. Exclusion for cardiogenic shock $(n=12)$ or multiple procedures yielded 99 retrospective cases for analysis. Because we were unable to gather retrospective data on certain variables, we prospectively tabulated 28 other traditional harvests done by the same team at other hospitals as a comparison group to determine harvest times, length of vein harvested, length of harvest incisions, and leg pain.

Statistical evaluation was aided by Systat 7.0 for Windows (Systat, Inc., Evanston, Ill.) with the Kruskal-Wallis test for nonparametric data and analysis of variance with the Bonferroni post hoc test for parametric data. Unless otherwise stated, all data are presented as mean values \pm the standard deviation. Additionally, several critical outcome variables were explored with more intensive statistical methods. For example, it might be expected that the length of the vein harvested and the length of the incision(s) for harvest were major contributors to leg complication rates and to leg pain. Multiple linear regression analyses, with interaction terms, were used for the scaled variable "leg pain at discharge," and multiple logistic regression analyses were used for categoric variables including presence or absence of any leg complication, presence or absence of leg infection, and presence or absence of any systemic complication. These analyses were preceded by univariate tests of all variables considered clinically applicable to a specific outcome. Variables tested are indicated by the superscripts in the appendix. We arbitrarily chose to include variables with a $p$ value of 0.15 or less in the initial multivariate model used to describe the outcome(s). A best-fit model was then ascertained by subsequent model testing with nonsignificant variables being dropped.

All comparisons in this study are between the 110 patients who underwent EVH versus the 99 patients who underwent the traditional procedure, with the exception of harvest times, leg pain, length of vein harvested, and length of incisions for harvest. In these areas, the EVH group is compared with the 28 patient prospective traditional group.

The variables tabulated for each patient, categorized by general area, are listed in the appendix.

Prophylactic cefazolin was used for all patients on the schedule of $1 \mathrm{gm}$ at operation, continued every 8 hours until 24 hours after chest tube removal. Vancomycin was substituted at $1 \mathrm{gm}$ per day if cefazolin was contraindicated.

Leg wound care was standardized. Patients showered and were told to wash the leg incisions with soap and water at day 2. If a patient was not ambulatory, the leg wounds were cleaned with normal saline solution every 8 hours and left open to air. If the wound was draining, it was cleaned as mentioned but covered with a dry dressing.

Pain assessments were made on a 0 (none) to 10 (worst imaginable) scale as determined by patient interview with the clinical nurse specialist at the indicated times.

EVH technique. After standard preparation, a transverse 2 to $3 \mathrm{~cm}$ incision was made at the medial aspect of the thigh $10 \mathrm{~cm}$ above the knee. The greater saphenous vein was identified, and an area over the vein was dis- sected to create a space that would allow the entry of a subcutaneous dissector.* A $300 \mathrm{~mm}$ 30-degree endoscope was inserted through a port in the dissector and the camera view was properly oriented.

The subcutaneous dissector was inserted into the space; by indirect vision via the video monitor, blunt dissection was initiated along the anterior surface of the vein. This was accomplished by holding the dissector tip just touching the surface of the vein and gently advancing the dissector with a slight side-to-side rocking motion to tease away the soft tissue over the vein. Simultaneously, an endoscopic miniscissor was used to perform sharp dissection along either side of the vein to further free the vein and to expose side branches. This minimized the chances of avulsing the branches. The combination of blunt and sharp dissection gave good exposure and an operative field largely free of blood.

A clip applier was advanced through the tunnel deep to the dissector, replacing the miniscissor. Large side branches were clipped proximally and divided several millimeters from the vein. Clips were not generally used on the harvest side. Small branches were generally not clipped and were divided without consequence.

This dissection process was carried to the saphenousfemoral junction. A vessel dissector was now placed into the tunnel. The C-shaped tip of the dissector was hooked around the vein and advanced toward the groin to separate the vein from the remaining soft tissue and to identify remaining undivided branches. The tip of the vessel dissector was occasionally used to retract the vein laterally to expose the remaining branches for clipping and dividing. Once carried to the femoral junction, this component of the harvest was completed.

This process was repeated distally toward and beyond the knee. Harvesting the segment of vein near the knee was occasionally tedious because of the numerous small branches and adhesions common to this area. A belowknee $3 \mathrm{~cm}$ transverse incision was used in 20 of 92 possible cases after our change to a single incision regimen to expedite the process.

With a single above-knee incision as described earlier, the vein was generally harvested proximally from the knee to the groin and distally from the knee to midcalf. If a greater length of vein was needed $(\geq 45 \mathrm{~cm})$, an additional small transverse incision was used distally (14 cases).

Finally, the vein was doubly clipped at the distal end with a $5 \mathrm{~mm}$ clip applier, divided, and delivered through the above-knee incision. Two Endoloop ligatures were applied over the free end of the vein and advanced to the femoral junction. The vein was doubly ligated and divided. The vein was then removed from the leg and prepared for grafting. The harvest site was inspected and, if satisfactory, the sutures securing the vein were trimmed and the incision(s) closed.

*All EVHs in this study used the Ethicon EVH kit (Ethicon Endo-Surgery, Inc., Cincinnati, Ohio) at a regionalized cost of $\$ 364.70$. An Ethicon Minishear device was also used at a regionalized cost of \$77.42. Ethicon Endoloop ligatures were used as the ligatures. 


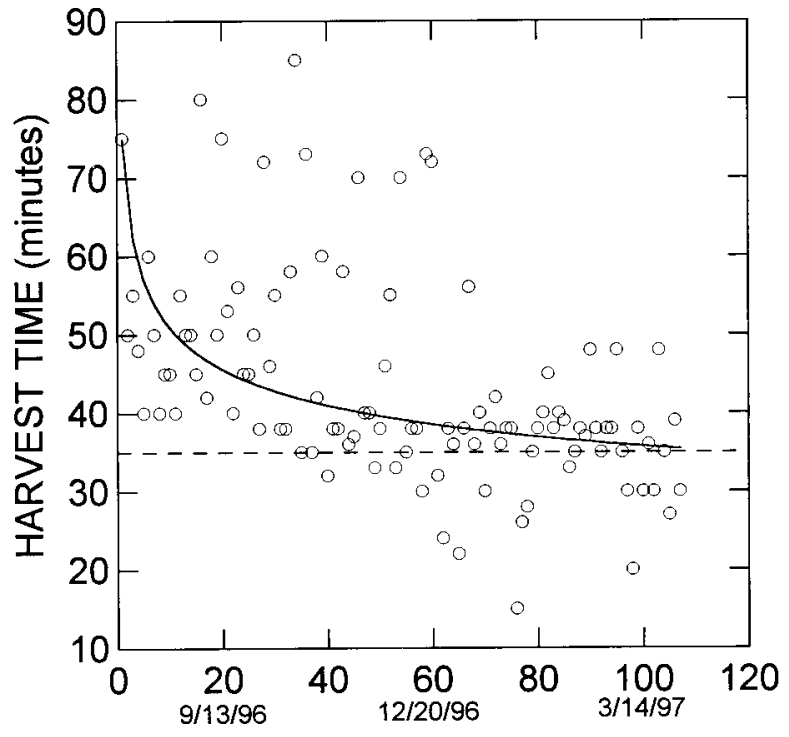

CASE PROGRESSION NUMBER

Fig. 1. Decrease in time required to harvest the saphenous vein as our experience increased. The abscissa is a simple numeric progression of our case experience: case 1 was performed on June 6, 1996; case 20, on September 13, 1996; case 40, on November 19, 1996; case 60, on December 20, 1996; case 80, on January 24, 1997; and case 100, on March 14, 1997. The line is an automated computer fit of a power function that appears to asymptote at about 35 minutes. The equation of the line is Harvest time $=79.93$ (case number $)^{-0.1718}$. Case 41 , with a harvest time of 125 minutes and included in the calculations and construction of the line, is not shown. The dashed line has been manually inserted at 35 minutes.

\section{Results}

Vein harvest variables. All veins in this series, except one traditionally harvested vein, were usable. Veins harvested endoscopically required $13.8 \pm 3.3$ branches to be clipped; veins harvested traditionally required $16.1 \pm 7.0$ branches to be clipped. Although the clipped number is higher for the traditional group $(p=0.035)$, we attribute this to the longer length of vein harvested in the traditional manner and of no significance. Leak repairs for the EVH veins totaled 29 (in 110 cases) and 8 (of 28 cases) for the traditionally harvested veins. These repair rates were not different $(p>0.20)$.

Our EVH procedure has evolved with experience. Initially, four small incisions were used. Incision sites were at the groin, above the knee, below the knee, and at the ankle. A single above-knee incision is now used. We believe that the elimination of the
Table I. EVH times versus experience

\begin{tabular}{lcrr}
\hline & First 15 cases & Last 15 cases & $p$ Value \\
\hline Vein harvest time & $53.5 \pm 15.0$ & $34.3 \pm 8.2$ & $<0.001$ \\
Closure time & $16.3 \pm 4.9$ & $9.0 \pm 4.5$ & $<0.001$ \\
Total time & $69.8 \pm 14.6$ & $53.9 \pm 6.0$ & $<0.001$ \\
\hline
\end{tabular}

Times are presented in minutes.

groin incision is important for reasons discussed later. This technique switch occurred very early on in our experience. The total length of all incisions for patients undergoing EVH was $5.7 \pm 3.1 \mathrm{~cm}$ (mid-tertile range, 3 to $7 \mathrm{~cm}$; median, $4 \mathrm{~cm}$ ). The traditional group incision showed a mean of $42.1 \pm$ $20.7 \mathrm{~cm}$ (mid-tertile, 36 to $44 \mathrm{~cm}$; median, $40 \mathrm{~cm}$; $p<0.001$ ). The length of vein harvested was $34.8 \pm$ $10.1 \mathrm{~cm}$ (mid-tertile, 30 to $38 \mathrm{~cm}$; median, $33 \mathrm{~cm}$ ) in the EVH group versus $45.5 \pm 21.3 \mathrm{~cm}$ (mid-tertile, 38 to $46 \mathrm{~cm}$; median, $43.5 \mathrm{~cm}$ ) in the traditional group $(p<0.001)$.

Harvest time in the EVH procedure showed a strong learning curve (Fig. 1 and Table I). From early harvests approximating 1 hour or more, the harvest time has dropped to what appears to be an asymptote at about 35 minutes. The abscissa of Fig. 1 is indicated as a case progression number, which simply assigned a numeric value to the cases in ascending order with the first case on June 6, 1996, and the last case on March 25, 1997. Overall, EVH harvests had a mean value of $44.3 \pm 15.9$ minutes versus traditional harvests at $25.0 \pm 9.6$ minutes $(p<0.001)$. Closure times were less $(p<0.001)$ for the EVH group (10.3 \pm 4.9 minutes) than for the traditional group (25.8 \pm 10.9 minutes). Total times (harvest plus closure) of $54.7 \pm 18.7$ minutes for the EVH group versus $50.8 \pm 19.1$ for the patients undergoing traditional harvest were not different $(p>0.20)$.

Outcomes variables. The hypothesis that EVH was equivalent to traditional harvest was tested by examination of broad outcomes areas including patient postoperative pain assessments and morbidities. Co-incident areas of study included the technical aspects of this procedure and practice changes resulting from EVH.

The patient populations were quite similar (Table II) with race, sex, type of insurance, age, height, weight, and number of bypasses required being equal. The American Society of Anesthesiologists $(p=0.003)$ and New York Heart Association $(p=$ $0.010)$ classifications were higher for the EVH group. Conversely, the predicted mortality rate (So- 
Table II. Selected patient demographics

\begin{tabular}{lccc}
\hline & Traditional & EVH & $p$ Value \\
\hline Age & $61.7 \pm 10.6$ & $61.8 \pm 10.2$ & $>0.20$ \\
Race & 94 White; 3 Asian; & 104 White; 2 Asian; & $>0.20$ (all comparisons) \\
Sex & 1 Hispanic; 1 black & 3 Hispanic; 1 black & $>0.20$ \\
Insurance type & $78 \mathrm{M} ; 21 \mathrm{~F}$ & $87 \mathrm{M} ; 23 \mathrm{~F}$ & $>0.20$ (all comparisons) \\
& 37 Medicare; 2 Medicaid; 58 private/ & 41 Medicare; 66 private/ & \\
Height $(\mathrm{cm})$ & corporate; 2 other & corporate; 3 other & $>0.20$ \\
Weight $(\mathrm{kg})$ & $174.0 \pm 10.5$ & $174.9 \pm 10.2$ & $>0.20$ \\
No. of distal anastomoses & $88.1 \pm 19.3$ & $86.1 \pm 17.5$ & $=0.160$ \\
ASA class & $3.3 \pm 1.0$ & $3.5 \pm 1.0$ & $=0.003$ \\
NYHA class & $1.76 \pm 0.63$ & $2.06 \pm 0.72$ & $=0.010$ \\
STS predicted deaths & $1.75 \pm 0.69$ & $2.00 \pm 0.63$ & $>0.20$
\end{tabular}

ciety of Thoracic Surgeons ${ }^{10,11}$ ) was equal between the groups: traditional $(2.51 \pm 3.00$; mid-tertile, 0.98 to 2.09; median, 1.43) versus EVH (2.21 \pm 2.51 ; mid-tertile 0.76 to 1.73 , median $=1.16), p>0.20$. Patients undergoing EVH had a greater smoking history (42 patients undergoing EVH were current smokers versus 16 patients undergoing traditional harvest; $p=0.004$ ) and more hypercholesterolemia (60 patients versus 39 patients; $p=0.034$ ). All other stratification factors, including the number of diseased coronary vessels $(\mathrm{EVH}, 2.6 \pm 0.67$, versus traditional, $2.4 \pm 0.76 ; p=0.053)$, distal anastomoses performed $(\mathrm{EVH}, 3.5 \pm 1.0$, versus traditional, $3.3 \pm 1.0 ; p=0.160$ ), and medications (appendix) were similar between the groups.

Pain assessments (Table III), comparing the 110 patients undergoing EVH with the prospective group of 28 patients undergoing traditional harvesting, at the time of discharge were $3.8 \pm 1.8$ (midtertile, 3 to 5 ; median, 3) for the patients undergoing traditional harvesting versus $0.1 \pm 0.4$ (mid-tertile, 0 ; median, 0$)$ for the patients undergoing $\operatorname{EVH}(p<$ $0.000)$. EVH pain assessments remained relatively constant and low to last follow-up $(0.2 \pm 0.6$; mid-tertile, 0 ; median, 0 ) at $0.9 \pm 0.04$ months. The traditional group pain assessments declined to $2.0 \pm$ 1.2 (mid-tertile, 2; median, 2) at the intermediate visit and $0.6 \pm 1.1$ (mid-tertile, 0; median, 0) at $1.3 \pm 0.1$ months at the final visit. Final pain assessments were not different $(p=0.092)$. Leg pain at the time of discharge from the hospital was also examined by multiple linear regression analyses. The type of harvest performed, the length of incisions made, and the length of vein harvested, along with the interactions between these three variables, were included. Even after an adjustment was made for the length of incisions and length of vein, the type of harvest remains the dominant correlate of pain $(p<0.000)$. The $p$ values for incision length, length of vein, and all interactions were all 0.113 or more.

Systemic morbidities were compared between the group of patients undergoing EVH and the group of 99 patients undergoing traditional harvesting. Taken over the course of this study, there were two superficial sternal infections and no deep sternal infections in the traditional group and no sternal infections in the EVH group. Two patients undergoing traditional harvesting became septic; no patients undergoing EVH became septic. Six patients undergoing traditional harvesting required prolonged ventilator support; one patient undergoing EVH required prolonged ventilator support. One patient from each group had a pulmonary embolus, and none had pulmonary edema. Pneumonia developed in one patient undergoing traditional harvesting and in two patients undergoing EVH; two patients undergoing traditional harvesting experienced respiratory distress syndrome versus one patient undergoing EVH. One patient undergoing traditional harvesting died and four patients undergoing EVH died, all directly related to the operative experience. None of these comparisons, taken individually, was statistically different $(p>0.20)$. Univariate logistic regression on several variables (appendix) was performed to determine which variables may have been contributory to systemic complications. Using the criteria presented in the Methods section of this article, the following variables were subsequently included in the initial multivariable logistic regression model: presence or absence of hypercholesterolemia, pulmonary hypertension, cardiomegaly, immune suppressor prescription drugs, atrial fibrillation, preoperative anticoagulant medication, preoperative antiplatelet medication, preoperative 
Table III. Selected outcomes

\begin{tabular}{lccc}
\hline & Traditional & EVH & p Value \\
\hline Pain at discharge (0 to 10 scale)* & $3.8 \pm 1.8$ & $0.1 \pm 0.4$ & $=0.000$ \\
Pain; 6-wk follow-up (0 to 10 scale)* & $0.6 \pm 1.1$ & $0.2 \pm 0.6$ & $=0.092$ \\
Hospital readmissions for leg complications (no.) & 3 & 1 & $>0.20$ \\
Surgeon office visits for leg complications (no.) & $56(15$ patients) & 18 (7 patients) & $<0.001$ \\
Vein harvest time (min)* & $25.0 \pm 9.6$ & $44.3 \pm 15.9$ & $<0.001$ \\
Harvest closure time (min)* & $25.8 \pm 10.9$ & $10.3 \pm 4.9$ & $<0.001$ \\
Total harvest time (min)* & $50.8 \pm 19.1$ & $54.7 \pm 18.7$ & $>0.20$ \\
Length of vein harvested (cm)* & $45.5 \pm 21.3$ & $34.8 \pm 10.1$ & $<0.001$ \\
Total length of harvest incisions (cm)* & $42.1 \pm 20.7$ & $5.7 \pm 3.1$ & $<0.001$ \\
Patient length of stay (days) & $6.3 \pm 5.3$ & $5.6 \pm 2.3$ & $=0.111$ \\
Length of surgery (min) & $218.0 \pm 55.5$ & $219.8 \pm 69.4$ & $>0.20$ \\
\hline
\end{tabular}

*Comparisons are drawn between the EVH group (110 patients) and the traditional prospective group (28) patients.

steroid medication, American Society of Anesthesiologists classification, New York Heart Association classification, the length of incision(s), the length of vein harvested, and leg pain at the time of discharge. Of particular note for variables excluded as noncontributory were the Society of Thoracic Surgeons predicted mortality, the admitting category, and the type of harvest procedure performed. Subsequent multiple logistic regression analyses eliminated all variables as noncontributory (all $p$ values $\geq 0.157$ ), except presence or absence of cardiomegaly and immune suppressor prescription drugs. With only the latter two variables in a model, the $p$ values were for 0.008 cardiomegaly and 0.006 for immune drugs. This latter model had an overall significance of 0.001 .

Leg morbidities (Table IV), compared between the 110 patients undergoing EVH and the 99 patients undergoing traditional harvesting, demonstrated the following results: In the immediate postoperative period, there were four leg infections in the traditional group and one leg infection in the EVH group. At 2 to 4 weeks after operation, there were two infections in the traditional group and three infections in the EVH group. At 6 weeks, two infections persisted in the patients who had undergone traditional harvesting, and all patients who had undergone $\mathrm{EVH}$ were clear (all comparisons $=p>$ $0.20)$.

There were 29 patients undergoing traditional harvesting and 26 patients undergoing EVH with edema at 2 to 4 weeks $(p>0.20)$. Other complications at this time (including wound separation, hematoma, seroma, cellulitis, necrosis, and/or drainage) totaled 28 for the patients undergoing traditional harvesting and 15 for the patients undergoing EVH $(p=0.051)$.
Later follow-up at approximately 6 weeks showed 14 cases of edema in the traditional group while 7 cases remained in the EVH group $(p>0.20)$. There remained, however, 21 other complications (as delineated earlier) in the patients undergoing traditional harvesting versus 3 in the EVH group $(p<$ $0.001)$.

Again, a more thorough logistic regression was used to determine which variables might have had an influence on the presence or absence of any leg complication. Variables were selected (appendix) and subjected to univariate logistic regression. Only the type of harvest was potentially contributory with $p=0.002$ and an odds ratio of 0.366 (95\% confidence interval bounds from 0.194 to 0.689 ).

A similar logistic procedure was used for the outcome of leg infection. Only the type of harvest procedure, sex, and the presence or absence of diabetes were indicated as potentially important in the univariate analyses. A multiple logistic regression model indicated that only the presence of diabetes was contributory to leg infections.

Three patients who had undergone traditional harvesting and one patient who had undergone $\mathrm{EVH}$ were readmitted for complications of vein harvest (Table III). The patient who had undergone EVH had a 5-day stay. Lengths of stay for two of the three patients who had undergone traditional harvesting were 23 hours and 4 days; the third patient was admitted for 9 days with discharge to a skilled nursing care facility for 3 days.

Fifteen patients were required to return to the surgeon's office for a total of 56 visits for leg wound complications in the traditional group (Table III). Seven patients for a total of 18 visits returned for leg wound care in the EVH group. The number of office visits required was significantly different $(p<0.001)$. 
The total surgery times (skin-to-skin) stemming from the harvest times delineated earlier were interesting. The EVH group showed a total surgery time of $219.8 \pm 69.4$ minutes (mid-tertile, 190 to 225 minutes; median, 207 minutes); the traditional group showed a mean of $218.0 \pm 55.5$ minutes (mid-tertile, 195 to 220 minutes; median, $210 \mathrm{~min}$ utes; $p>0.20$ ).

Length of stay from surgery to discharge was not different between the patient groups. Patients who had undergone traditional harvesting were discharged at $6.3 \pm 5.3$ days (mid-tertile, 4 to 5 days; median, 4 days) after surgery; patients who had undergone EVH were discharged at $5.6 \pm 2.3$ days (mid-tertile, 4 to 6 days; median, 5 days) after operation $(p=0.111)$.

Differences in day of ambulation between the groups were demonstrable. The EVH group were ambulatory at day $2.1 \pm 1.0$ versus the traditional group who were ambulatory at day $1.6 \pm 0.7(p=$ $0.005)$.

\section{Discussion}

Our data clearly showed that patient pain as determined by patient declaration was significantly reduced with the EVH versus the traditional harvest and is consistent with earlier reports. ${ }^{6,9}$ This benefit was apparent for several weeks. The low pain level in the patients who had undergone EVH was so marked that patients expressed surprise when asked about their leg pain.

In the test of a new procedure, one of the standards of comparison is whether the new procedure accomplishes the goal with, at least, no increase in morbidity. Ideally, a decrease in complications would result from the new technique. Such was indeed the case for EVH in our hands. Overall systemic morbidities were equal, and leg morbidities were equal or better for EVH in all parameters measured. The multiple logistic regression analyses gave strong evidence that the type of harvest procedure, length of incisions made, and length of vein taken did not contribute to the presence of any systemic complication. The multiple linear regression analyses further showed that only the type of harvest procedure performed was contributory to leg pain at discharge, with length of vein taken, incision length, and their interactions being noncontributory. Finally, logistic regression clearly demonstrated that endoscopic harvesting did not contribute to increased leg infections and was highly favorable for lessened overall leg complications.
Table IV. Morbidities

\begin{tabular}{lccc}
\hline & Traditional & EVH & p Value \\
\hline $\begin{array}{l}\text { Leg infections at 2- to } \\
\text { 4-week visit }\end{array}$ & 2 & 3 & $>0.20$ \\
$\begin{array}{l}\text { Leg edema at 2- to } \\
\quad \text { 4-week visit }\end{array}$ & 29 & 26 & $>0.20$ \\
$\begin{array}{c}\text { Total other leg com- } \\
\text { plications at 2- to } \\
\text { 4-week visit* }\end{array}$ & 28 & 15 & $=0.051$ \\
$\begin{array}{l}\text { Leg infections at } \\
\text { 6-week visit }\end{array}$ & 2 & 0 & $>0.20$ \\
$\begin{array}{c}\text { Leg edema at 6-week } \\
\text { visit }\end{array}$ & 14 & 7 & $>0.20$ \\
$\begin{array}{c}\text { Total other leg com- } \\
\text { plications at 6-week }\end{array}$ & 21 & 3 & $<0.001$ \\
visit* & & &
\end{tabular}

*See text for complication listing.

The economic consequences of the morbidities that did occur again strongly favor the EVH over the traditional procedure. One patient who had EVH returned to hospital for leg wound care versus three patients for the traditional group. Office visits were required for leg wound care by more than $13 \%$ of patients who underwent traditional harvesting, with a total of 56 visits. This contrasts to the $6.3 \%$ return (with a total of 18 visits) for the EVH group. The overall economic benefits are obvious, including the use of human resources. In fact, the low leg wound complication rate in the patients who underwent EVH prompted a change in our algorithm of care for patients undergoing coronary bypass. An intermediate visit has been eliminated with the patients now being seen at approximately 2 weeks after operation and again 2 to 4 weeks later for surgical discharge.

The length of vein harvested was fully adequate for our technique of sequential anastomoses. If a longer length of vein is necessary, the addition of a second small incision at mid-calf allows a full-length vein harvest for those surgeons who use individual grafts.

Minimally invasive harvest times have decreased steadily as our experience has grown. It would seem that a baseline of about 35 minutes for harvest is reasonable. This is still about 10 minutes longer than an open harvest. If one adds harvest site closure time to the equation, then the two techniques are similar. If the surgeon has a first assistant (not the harvester) and is therefore not delayed by the closure, then inclusion of closure time in the harvest equation may be of no consequence. If, however, the 
vein harvester is also the first assistant, then the $\mathrm{EVH}$ is comparable to the traditional open harvest. Our procedure was that the surgeon opened the chest, harvested an internal thoracic artery, and prepared for and initiated cardiopulmonary bypass concurrently with the vein harvest. Because we have demonstrated that EVH does not contribute to an increase in operating time, this practice has proved to be efficient.

Both groups of patients were ambulatory (walking in the hallway) on postoperative day 2. This is a result of the algorithm of care used by the hospitals and is independent of the vein harvest technique.

\section{Summary}

EVH, even in its evolutionary phase, is a desirable procedure and will very likely become the vein harvest procedure of choice. It is less painful for the patient; it carries fewer morbidities; it is likely to be much less costly overall, and it accomplishes the goal of adequate vein harvest for coronary bypass in a reasonable time by a single operator. It is effective, safe, and financially prudent.

\section{REFERENCES}

1. Lee KS, Reinstein L. Lower limb amputation of the donor site extremity after coronary artery bypass graft surgery. Arch Phys Med Rehabil 1986;67:564-5.

2. DeLaria GA, Hunter JA, Goldin MD, Serry C, Javid H, Najafi H. Leg wound complications associated with coronary revascularization. J Thorac Cardiovasc Surg 1981;81:403-7.

3. Baddour LM, Bisno AL. Recurrent cellulitis after coronary bypass surgery. JAMA 1984;251:1049-52.

4. Utley JR, Thomason ME, Wallace DJ, et al. Preoperative correlates of impaired wound healing after saphenous vein excision. J Thorac Cardiovasc Surg 1989;98:147-9.

5. Wipke-Tevis DD, Stotts NA, Skov P, Carrieri-Kohlman V. Frequency, manifestations, and correlates of impaired healing of saphenous vein harvest incisions. Heart Lung 1996;25: 108-16.

6. Rashid A, Fabri B, Meade JB. Subcutaneous technique for saphenous vein harvest. Ann Thorac Surg 1984;37:169-70.

7. Cusimano RJ, Dale L, Butany JW. Minimally invasive cardiac surgery for removal of the greater saphenous vein. Can J Surg 1996;39:386-8.

8. Lumsden AB, Eaves FF III, Ofenloch JC, Jordan WD. Subcutaneous, video-assisted saphenous vein harvest: report of the first 30 cases. Cardiovasc Surg 1996;6:771-6.

9. Tevaearai HT, Mueller XM, von Segesser LK. Minimally invasive harvest of the saphenous vein for coronary artery bypass grafting. Ann Thorac Surg 1997;63:S119-21.

10. Clark RE. The Society of Thoracic Surgeons national data base status report. Ann Thorac Surg 1994;57:20-6.

11. Clark RE. The STS cardiac surgery national database: an update. Ann Thorac Surg 1995;59:1376-80.

\section{Appendix. Database variables}

Superscripts denote those variables used in univariate analyses for leg pain at discharge, ${ }^{\mathrm{a}}$ any systemic complication, ${ }^{\mathrm{b}}$ any leg complication, ${ }^{\mathrm{c}}$ or postoperative leg infection. $^{\mathrm{d}}$

Administrative

Data entry person and date

Case number

Account number

Patient date of birth ${ }^{\mathrm{b}, \mathrm{c}, \mathrm{d}}$

Patient sex ${ }^{\mathrm{b}, \mathrm{c}, \mathrm{d}}$

Patient race

Surgical team information

Surgeon

First assistant

Vein harvester

Stratification factors

Current smoker ${ }^{\mathrm{b}, \mathrm{c}, \mathrm{d}}$

Family history of coronary artery disease

Diabetes ${ }^{\mathrm{b}, \mathrm{c}, \mathrm{d}}$

Obesity $^{\mathrm{b}, \mathrm{c}, \mathrm{d}}$

Hypercholesterolemia ${ }^{\text {b,c,d }}$

Renal failure ${ }^{\mathrm{b}}$

Patient on dialysis

Hypertension $^{\mathrm{b}}$

Pulmonary hypertension ${ }^{\mathrm{b}}$

History of cerebral vascular accident

Endocarditis ${ }^{\mathrm{b}}$

Cardiomegaly $^{\mathrm{b}}$

Chronic obstructive pulmonary disease ${ }^{\mathrm{b}}$

Peripheral vascular disease $\mathrm{e}^{\mathrm{b}, \mathrm{c}, \mathrm{d}}$

Cerebral vascular disease ${ }^{\mathrm{b}}$

Operative incident for bypass ${ }^{\mathrm{b}}$

History of percutaneous transluminal coronary angioplasty (PTCA)

History of atherectomy

History of stent placement

History of thrombolysis

History of a previous myocardial infarction

Procedure defined as an emergency after PTCA

History of valvuloplasty

Presence of congestive heart failure ${ }^{\mathrm{b}, \mathrm{c}, \mathrm{d}}$

Unstable angina

Stable angina

Patient in cardiogenic shock

Patient had been resuscitated before this procedure

Arrhythmia (acute or chronic)

Ventricular $^{\mathrm{b}}$

Atrial fibrillation ${ }^{\mathrm{b}}$

Atrioventricular block

Complete heart block

Patient symptomatic

American Society of Anesthesiologists classification ${ }^{\mathrm{b}}$

New York Heart Association classification ${ }^{\mathrm{b}}$

Society of Thoracic Surgeons Predicted Mortality

Presence of aortic valve disease

Presence of mitral valve disease

Admitting category ${ }^{\mathrm{b}, \mathrm{c}, \mathrm{d}}$

Number of diseased coronary vessels

Presence of left main disease ${ }^{b}$

Varicose veins ${ }^{\mathrm{c}, \mathrm{d}}$

Preoperative medications ${ }^{\mathrm{b}(\mathrm{all})}$ 
Digitalis

Calcium antagonists

Nitrates by mouth ${ }^{\mathrm{c}, \mathrm{d}}$

Nitrates, intravenous

Antiarrhythmics

Anticoagulants ${ }^{\mathrm{c}, \mathrm{d}}$

Inotropic drugs

Aspirin $^{\mathrm{c}, \mathrm{d}}$

Beta-blockers

Angiotensin-converting enzyme inhibitors

Antiplatelet drugs

Diuretics $^{\mathrm{c}, \mathrm{d}}$

Steroids

Immunosuppresive prescription drugs

Warfarin sodium (Coumadin)

Operative data

Type of harvest procedure $\mathrm{a}^{\mathrm{a}, \mathrm{b}, \mathrm{c}, \mathrm{d}}$

Number of distal anatomoses performed

Time required for surgery

Were inotropic agents used in operating room?

Were antiarrhythmics used in operating room?

Was excessive bleeding present at operation?

Did any of the grafts occlude acutely?

Was there an intraoperative myocardial infarction?

Specific vein harvest operative information

Was preoperative mapping used for the vein harvest site

Were any of the minimally invasive harvests converted to open

If so, what was the reason?

Number of incisions used for harvest

Total length of incisions used ${ }^{\mathrm{a}, \mathrm{b}, \mathrm{c}, \mathrm{d}}$

Location of starting point for harvest

Length of vein harvested ${ }^{\mathrm{a}, \mathrm{b}, \mathrm{c}, \mathrm{d}}$

Was the vein suitable for bypass grafting?

Number of side branches clipped

Number of leaks repaired

Harvest time

Closure time
Were iatrogenic side tears made in the vein?

Were other injuries made on the vein?

Was leg bleeding excessive ( $>25 \mathrm{ml}$ by harvester estimate)?

Did the vein bifurcate abnormally?

Did the vein have nonstandard anatomy?

Postoperative information and follow-up

Did a superficial sternal infection develop?

Did a deep sternal infection develop?

Did a leg (harvest site) infection develop?

Did the patient become septic?

Was prolonged ventilator support required?

Did a pulmonary embolus occur?

Was pulmonary edema present?

Did pneumonia develop?

Did the respiratory distress syndrome develop?

Did the patient die?

If so, when and what was the cause?

Was the patient readmitted within 30 days of the procedure?

If so, when and what was the cause?

What day did the patient become ambulatory after the operation?

What was the patient declaration of leg pain at discharge? ${ }^{\mathrm{b}}$

Vein postoperative information (taken within 1 week, at 2 to

4 weeks, and at last follow-up)

Did wound separation occur?

Was there a postoperative hematoma?

Did cellulitis develop?

Did a seroma develop?

Was necrosis present?

Did an infection develop?

Was excessive edema present?

Did the wound drain?

Pain assessments

How many office visits were required for leg wound care?

Was the patient readmitted for leg wound care? 